\title{
An Ultrastructural Characterization of the Ooplasm in Ovarian Follicles of the Immature Ostrich (Struthio camelus)
}

\author{
M.-C. Madekurozwa and W. H. Kimaro \\ Address of authors: Department of Anatomy and Physiology, Faculty of Veterinary Science, University \\ of Pretoria, Private bag X04, Onderstepoort 0110, South Africa ; Corresponding author: Tel.: +27 12 \\ 529 8417; e-mail: madex@op.up.ac.za \\ Correspondence to Department of Anatomy and Physiology, Faculty of Veterinary Science, University \\ of Pretoria, Onderstepoort, South Africa
}

\begin{abstract}
Primordial, pre-vitellogenic and vitellogenic follicles were present in the ovary of the immature ostrich. The oocytes of these follicles were composed of a nucleus surrounded by ooplasm. Central, intermediate and cortical regions formed the ooplasm. The organelles present in these ooplasmic regions varied depending on the stage of follicular development. In primordial and small pre-vitellogenic (100-150 $\mu \mathrm{m}$ in diameter) follicles the central region of the ooplasm was dominated by an accumulation of organelles, which formed Balbiani's vitelline body. In contrast, the central region in vitellogenic follicles was filled with numerous large yolk spheres, many of which contained lining bodies. Numerous lipid droplets interspersed with mitochondria and small yolk spheres formed the intermediate ooplasmic region in primordial and small pre-vitellogenic follicles. In large pre-vitellogenic (150-400 $\mu \mathrm{m}$ in diameter) and vitellogenic follicles the intermediate region contained a greater density of mitochondria and small yolk spheres. Small yolk spheres were observed in the cortical region of pre-vitellogenic follicles. An interesting feature of the cortical region in vitellogenic follicles was the frequent occurrence of Golgi complexes. The results of the study indicate that although the ovarian follicles in the immature ostrich are not ovulated, the components and composition of the ooplasm are similar to those observed in the mature follicles of other avian species.
\end{abstract}

\section{Introduction}

Previous ultrastructural and immunohistochemical studies on the ovary of the immature ostrich (12-14 months old) have demonstrated the occurrence of cyclical reproductive activity, which appears to be influenced by photoperiod (Kimaro and Madekurozwa, 2006; Madekurozwa and Kimaro, 2006a,b). During periods of short day-length, the immature ostriches contain inactive ovaries, while active ovaries are present during periods of longer day-lengths (Kimaro and Madekurozwa, 2006). The inactive ovary is a flattened structure, which has a granular appearance on account of the presence of numerous follicles, which are less than $2 \mathrm{~mm}$ in diameter. In contrast, the active ovary contains predominantly yellow yolkfilled follicles, with the diameters of the largest follicles ranging from 11 to $19 \mathrm{~mm}$ (Madekurozwa and Kimaro, 2006a). Ostriches generally become sexually mature at 24 years of age (Hicks-Alldredge, 1998). Thus, the follicles in immature birds (12-14 months old) are not ovulated, but instead undergo atresia (Madekurozwa and Kimaro, 2006a). Lightand electron-microscopic studies have described the morphology of ovarian follicles in the immature ostrich (Madekurozwa and Kimaro, 2006a,b). However, the ultrastructural features of the ooplasm in these ovarian follicles have not been elucidated. To date, most studies on the ooplasm have been conducted in sexually mature birds (Bellairs, 1965, 1967; Perry and Gilbert, 1979; Ito et al., 2003; Zarnescu, 2004). Relatively little is known about the components of the ooplasm in immature birds. The purpose of the present study was to describe changes in the features of the ooplasm associated with the maturation of follicles in the immature ostrich. The results of the study will provide further information on the precocious reproductive activity of the immature ostrich. 


\section{Materials and Methods}

A total of 26 sexually immature female ostriches with active ovaries were used in the present study. The birds, which were bred in commercial farms, were aged between 12 and 14 months, and weighed $90-100 \mathrm{~kg}$. At a commercial abattoir, the ostriches were electrically stunned and exsanguinated. Ovarian tissue was immersion-fixed in $2.5 \%$ glutaraldehyde in $0.1 \mathrm{~m}$ cacodylate buffer ( $\mathrm{pH}$ 7.3). Thereafter, the tissue samples were post-fixed in osmium tetroxide, dehydrated and embedded in epoxy resin. Ultrathin sections were stained with lead citrate and uranyl acetate. The samples were viewed with a Philips CMIO transmission electron microscope (FEI, Eindhoven, the Netherlands).

\section{Results}

Primordial, pre-vitellogenic and vitellogenic follicles were identified in the ovaries of the immature ostriches. Primordial follicles were composed of an oocyte surrounded by a layer of flat granulosa cells. The granulosa cells were enclosed in a single layer of squamous thecal cells. A simple columnar or pseudo-stratified columnar granulosa cell layer surrounded the oocyte in pre-vitellogenic follicles. The thecal layer in pre-vitellogenic follicles was undifferentiated. A simple cuboidal or columnar cell layer surrounded the oocyte in vitellogenic follicles. The thecal layer in these follicles was clearly differentiated into theca interna and theca externa. In all follicles the oocyte contained a nucleus surrounded by ooplasm. Cortical, intermediate and central regions formed the ooplasm.

\section{Primordial follicles}

In primordial follicles intercellular spaces $(0.2 \mu \mathrm{m}$ wide) separated the granulosa cells. The intercellular spaces contained an amorphous electron-dense material. The oolemma in contact with the amorphous material exhibited several pinocytotic vesicles (Fig. 1a). Occasional microvillous processes extended from the apical region of the granulosa cells. Lining bodies coated the apices of the microvillous processes (Fig. 1a,b). The lining bodies consisted of three electron-dense layers, with the inner and middle, as well as the middle and outer layers separated by electron-lucent areas. The inner layers of the lining bodies exhibited electron-dense granules.

In early primordial follicles lipid droplets dominated the cortical and intermediate regions of the ooplasm. In the later stages of primordial follicle development the cortical region of the ooplasm contained microfilaments, vesicles, electron-dense bodies and a few mitochondria. The intermediate ooplasmic region, which was situated deep in the cortical layer, contained numerous lipid droplets (Fig. 1a). Mitochondria and occasional small yolk spheres (0.3$0.5 \mu \mathrm{m}$ in diameter) were interspersed between the lipid droplets. Some of the small yolk spheres contained lining bodies (Fig. 1C).

The central region of the ooplasm contained a nucleus and Balbiani's vitelline body (Fig. 2a). Balbiani's vitelline body was composed of a dense accumulation of organelles and inclusions, which included mitochondria, Golgi complexes, vesicles, lipid droplets, rough endoplasmic reticulum (RER) profiles and annulate lamellae (Fig. 2b,c). The annulate lamellae were composed of concentrically arranged membranous profiles (Fig. 2c). Several vesicles were seen in close proximity to the annulate lamellae.

\section{Pre-vitellogenic follicles}

The cortical region of the ooplasm in small pre-vitellogenic follicles (100-150 $\mu \mathrm{m}$ in diameter) contained RER profiles, free ribosomes, vesicles and relatively few mitochondria. At this stage, a well-defined Balbiani's vitelline body, composed of numerous mitochondria and several Golgi complexes, was still situated in the central region of the oocyte. The intermediate region of the ooplasm contained numerous lipid droplets and clumps of mitochondria. 
The components of Balbiani's vitelline body dispersed to the cortical and intermediate ooplasmic regions in large pre-vitellogenic follicles (150-400 $\mu \mathrm{m}$ in diameter). The cortical region of the ooplasm in these pre-vitellogenic follicles contained mitochondria, vesicles and small yolk spheres (Fig. 3a). Located within the small yolk spheres were well-defined lining bodies (Fig. 3b). The intermediate region of the ooplasm contained numerous mitochondria and small yolk spheres. In addition, a few lipid droplets were observed (Fig. 3a). The central region of the ooplasm typically contained numerous lipid droplets (Fig. 3c). Fewer small yolk spheres occurred in this central region.

\section{Vitellogenic follicles}

The plasma membranes of both the oocyte and granulosa cell layer were thrown into microvilli, resulting in the formation of the zona radiata. Numerous lining bodies were exhibited on the apices of microvilli, which extended from the granulosa cells (Fig. 4a). Mitochondria and vesicles dominated the cortical region of the ooplasm. In addition, Golgi complexes were frequently observed in this region (Fig. $4 b$ ).

The intermediate region of the ooplasm contained small yolk spheres, vesicles and mitochondria. Larger yolk spheres, which were at a more advanced stage of development than the small yolk spheres, occurred in the central region of the ooplasm (Fig. 5a). Large yolk spheres varied greatly in size (1-2.5 $\mu \mathrm{m}$ in diameter) and stage of development. The larger yolk spheres contained several lining bodies (Fig. 5a) and spherical electron-dense yolk granules (Fig. 5b). Surrounding the yolk granules and lining bodies was a granular, electron-dense material. Interspersed between the large yolk spheres were small yolk spheres, mitochondria, vesicles, Golgi complexes and lipid droplets. Although most of the larger yolk spheres contained intact lining bodies, degenerating lining bodies were occasionally observed (Fig. 5c).

\section{Discussion}

Most research on the ooplasm has been conducted on the domestic fowl (Bellairs, 1965, 1967; Greenfield, 1966). In this avian species, lipid droplets dominate the entire ooplasm in the early oocyte (Bellairs, 1965, 1967). This stage of development corresponded to the early phase of primordial follicle development observed in the immature ostrich. In the later stages of primordial follicle development in the immature ostrich, the cortical region of the ooplasm was devoid of lipid droplets. This stage of development is considered to be the second phase of oocyte maturation in the domestic fowl (Bellairs, 1965).

An interesting feature of the oocyte in primordial follicles is the presence of a dense accumulation of organelles, known as Balbiani's vitelline body, adjacent to the nucleus. Balbiani's vitelline body typically contains numerous mitochondria interspersed with Golgi complexes and vesicles (Greenfield, 1966; Bellairs, 1967). Occasional annular lamellae were identified in the Balbiani's vitelline body of the immature ostrich. The location and constituents of Balbiani's vitelline body appear to be consistent across avian species (Greenfield, 1966; Bellairs, 1967). With the progression of follicular development, the organelles forming Balbiani's vitelline body disperse to the cortical and intermediate regions of the ooplasm, which are the sites of yolk formation (Greenfield, 1966; Bellairs, 1967). In the immature ostrich, the dispersal of the organelles forming Balbiani's vitelline body began in large pre-vitellogenic follicles and was completed during the vitellogenic follicular stage. A feature common to the domestic fowl (Bellairs, 1965, 1967) and the immature ostrich, at the vitellogenic stage of follicular development, was the formation of lining bodies on microvillous processes extending from granulosa cells. Lining bodies are membranous organelles involved in the transportation of the yolk precursor vitellogenin into the oocyte (Ito et al., 2003). Vitellogenin is one of the major yolk precursors synthesized by the liver (Deeley et al., 1975). On account of the controversy surrounding the origin, function and fate of lining bodies, these structures are known by several terms including, 'transosomes' (Schjeide et al., 1974), 'unique bodies' (Schjeide et al., 1966), 'coated vesicles' (Perry and Gilbert, 1979) and 'terminal membranes' (Wyburn et al., 1965a). Lining bodies have been observed in the domestic fowl (Bellairs, 1965; Wyburn et al., 1965a,b; Greenfield, 1966; Schjeide et al., 1966), Japanese quail (Ito et al., 2003), dove (Zarnescu, 2004) and ostrich (Madekurozwa and Kimaro, 2006b). In the domestic fowl (Paulson and Rosenberg, 1972), Japanese quail (Ito et al., 2003) and ostrich (Madekurozwa and Kimaro, 2006b), lining bodies appear to originate from the lateral plasma membranes of adjacent granulosa cells. They 
then migrate from the lateral to the apical plasma membranes of the granulosa cells, where they are attached to microvilli. The occurrence of lining bodies on the tips of granulosa cell microvilli is well documented (Schjeide et al., 1966, 1974; Paulson and Rosenberg, 1972; Ito et al., 2003; Madekurozwa and Kimaro, 2006b). The lining bodies are subsequently engulfed by the oocyte, and then incorporated into yolk spheres (Ito et al., 2003).

The morphology of large yolk spheres in the immature ostrich appeared to be identical to those described in the sexually mature domestic fowl (Bellairs, 1967; Paulson and Rosenberg, 1972), Japanese quail (Ito et al., 2003) and dove (Zarnescu, 2004). As in these species, the large yolk spheres in the immature ostrich contained yolk granules and fine electron-dense particles in addition to the lining bodies. Yolk granules in the Japanese quail have been shown to contain lipovitellin and phosvitin, which are products resulting from the enzymatic cleavage of vitellogenin (Deeley et al., 1975; Elkin et al., 1995). Cathepsin D, the enzyme responsible for the cleavage of vitellogenin has been localized in the yolk granules of the domestic fowl (Shen et al., 1993). Immunohistochemical studies need to be carried out on the yolk granules of the immature ostrich to determine their biochemical composition.

In addition to vitellogenin, the liver produces a yolk precursor known as very low-density lipoprotein (Barber et al., 1991). It is generally accepted that very low-density lipoprotein, produced in the liver and transported in the blood stream, reaches the oocyte by traversing the basal lamina and the intercellular spaces between granulosa cells (Jordanov and Boyadjieva-Michailova, 1974;Evans et al., 1979; Perry et al., 1984; Barber et al., 1991). This argument is supported by the findings of the present study, in which an amorphous material was observed in the intercellular spaces of granulosa cells in primordial follicles. Jordanov and Boyadjieva-Michailova (1974) traced the movement of lipoprotein through the intercellular spaces between granulosa cells to the oocyte. It was suggested that the lipoprotein reached the ooplasm by passing through pores in the oolemma. However, further research conducted by Shen et al. (1993) demonstrated the presence of very low-density lipoprotein in vesicles attached to the oolemma. Similar observations were made in the primordial follicles of the immature ostrich. Researchers have further clarified that the endocytotic process involved in the transfer of very lowdensity lipoprotein into the oocyte is receptor-mediated.

In conclusion, the results of the present study have shown that the components of the ooplasm in developing follicles in the immature ostrich are similar to those of other avian species. Further research is required to determine the biochemical composition of large yolk spheres and lining bodies in the ostrich.

\section{Acknowledgements}

The authors thank staff in the Electron Microscope Unit and the Department of Education Innovation (Creative Studios) for their technical assistance. The University of Pretoria and the National Research Foundation (Thuthuka programme) funded this study.

\section{References}

- Barber, D.L., E.J. Sanders, R. Aebersold, and W.J. Schneider, 1991: The receptor for yolk lipoprotein deposition in the chicken oocyte. J. Biochem. Chem. 28, 1876118770.

- Bellairs, R., 1965: The relationship between oocyte and follicle in the hen's ovary as shown by electron microscopy. J. Embryol. Exp. Morph. 13, 215-233.

- Bellairs, R., 1967: Aspects of the development of yolk spheres in the hen's oocyte, studied by electron microscopy. J. Embryol. Exp. Morph. 17, 267-281.

- $\quad$ Deeley, R.G., K.P. Mullinix, W. Wetekam, H.M. Kronenberg, M. Meyers, J.D. Eldridge, and R.F. Goldberger, 1975: Vitellogenin synthesis in the avian liver. J. Biol. Chem. 250, 9060-9066.

- $\quad$ Elkin, R.G., M.B. Freed, S.A.H. Danetz, and C.A. Bidwell, 1995: Proteolysis of Japanese quail and chicken plasma apolipoprotein $B$ and vitellogenin by cathepsin $D$ : similarity of the resulting protein fragments with egg yolk polypeptides. Comp. Biochem. Physiol. 112B, 191-196. 
- $\quad$ Evans, A.J., M.M. Perry, and A.B. Gilbert, 1979: The demonstration of very low density lipoprotein in the basal lamina of the granulosa layer in the hen's ovarian follicle. Biochim. Biophys. Acta 573, 184-195.

- Greenfield, M.L., 1966: The oocyte of the domestic chicken shortly after hatching, studied by electron microscopy. J. Embryol. Exp. Morph. 15, 297-316.

- $\quad H i c k s-A l l d r e d g e$, K., 1998: Ratite reproduction. Vet. Clin. N. Am. 14, 437-453.

- Ito, Y., M. Kihara, E. Nakamura, S. Yonezawa, and N. Yoshizaki, 2003: Vitellogenin transport and yolk formation in the quail ovary. Zool. Sci. 20, 717-726.

- Jordanov, J., and A. Boyadjieva-Michailova, 1974: Ultrastructural aspects of lipoprotein passage through oocyte envelopes and storage in ooplasm during avian vitellopoiesis. Acta Anat. 89, 616-632.

- Kimaro, W.H., and M.-C. Madekurozwa, 2006: Immunoreactivities to protein gene product 9.5, neurofilament protein and neuron specific enolase in the ovary of the sexually immature ostrich (Struthio camelus). Exp. Brain Res. 173, 291-297.

- Madekurozwa, M.-C., and W.H. Kimaro, 2006a: A morphological and immunohistochemical study of healthy and atretic follicles in the ovary of the sexually immature ostrich (Struthio camelus). Anat. Histol. Embryol. 35, 253-258.

- Madekurozwa, M.-C., and W.H. Kimaro, 2006b: Ultrastructural features of the follicular wall in developing follicles of the sexually immature ostrich (Struthio camelus). Onder. J. Vet. Res. 73, 199-205.

- Paulson, J., and M.D. Rosenberg, 1972: The function and transposition of lining bodies in developing avian oocytes. J. Ultrastructure Res. 40, 25-43.

- Perry, M.M., and A.B. Gilbert, 1979: Yolk transport in the ovarian follicle of the hen (Gallus domesticus): lipoprotein-like particles at the periphery of the oocyte in the rapid growth phase. J. Cell Sci. 39, 257-272.

- $\quad$ Perry, M.M., H.D. Griffin, and A.B. Gilbert, 1984: The binding of very low density lipoproteins to the plasma membrane of the hen's oocyte. A morphological study. Exp. Cell Res. 151, 433-446.

- $\quad$ Schjeide, O.A., R.J. Munn, R.G. McCandless, and R. Edwards, 1966: Unique organelles of avian oocytes. Growth 30, 471-489.

- Schjeide, O.A., L. Hanzely, S.J. Holshouser, and W.E. Briles, 1974: Production and fate of unique organelles (transosomes) in ovarian follicles of Gallus domesticus under various conditions. Cell Tissue Res. 156, 47-59.

- $\quad$ Shen, X., E. Steyrer, H. Retzek, E.J. Sanders, and W.J. Schneider, 1993: Chicken oocyte growth: receptor-mediated yolk deposition. Cell Tissue Res. 272, 459-471.

- Wyburn, G.M., H.S. Johnston, and R.N.C. Aitken, 1965a: Specialised plasma membranes in the preovulatory follicle of the fowl. Zeit. Zell. 68, 70-79.

- Wyburn, G.M., R.N.C. Aitken, and H.S. Johnston, 1965b: The ultrastructure of the zona radiata of the ovarian follicle of the domestic fowl. J. Anat. 99, 469-484.

- Zarnescu, O., 2004: Ultrastructural observations of previtellogenic ovarian follicles of dove. Zygote 12, 285-292. 

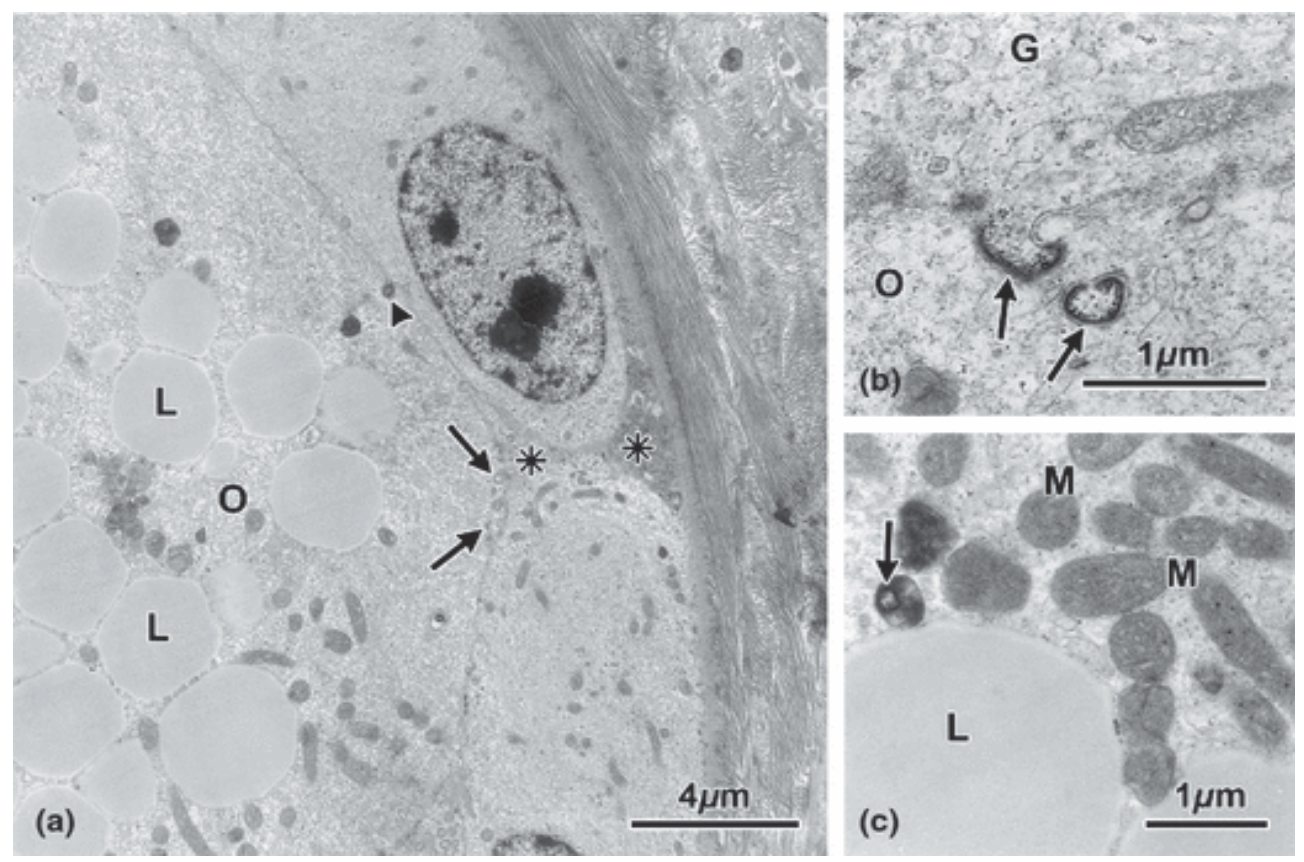

Fig. 1. (a) Granulosa cells and oocyte (O) of a primordial follicle. The intercellular space between the granulosa cells is filled with an amorphous substance (asterisks). Pinocytotic vesicles (arrows) protrude into the ooplasm. Occasional lining bodies (arrowhead) are present. Numerous lipid droplets $(L)$ are seen in the intermediate region of the ooplasm. (b) Microvillous of a granulosa cell (G) coated with a lining body (arrows). The innermost layer of the lining body exhibits electron-dense granules. O: oocyte. (c) Small yolk sphere with an enclosed lining body (arrow) in the intermediate region of the ooplasm. M: mitochondria. L: lipid droplet.

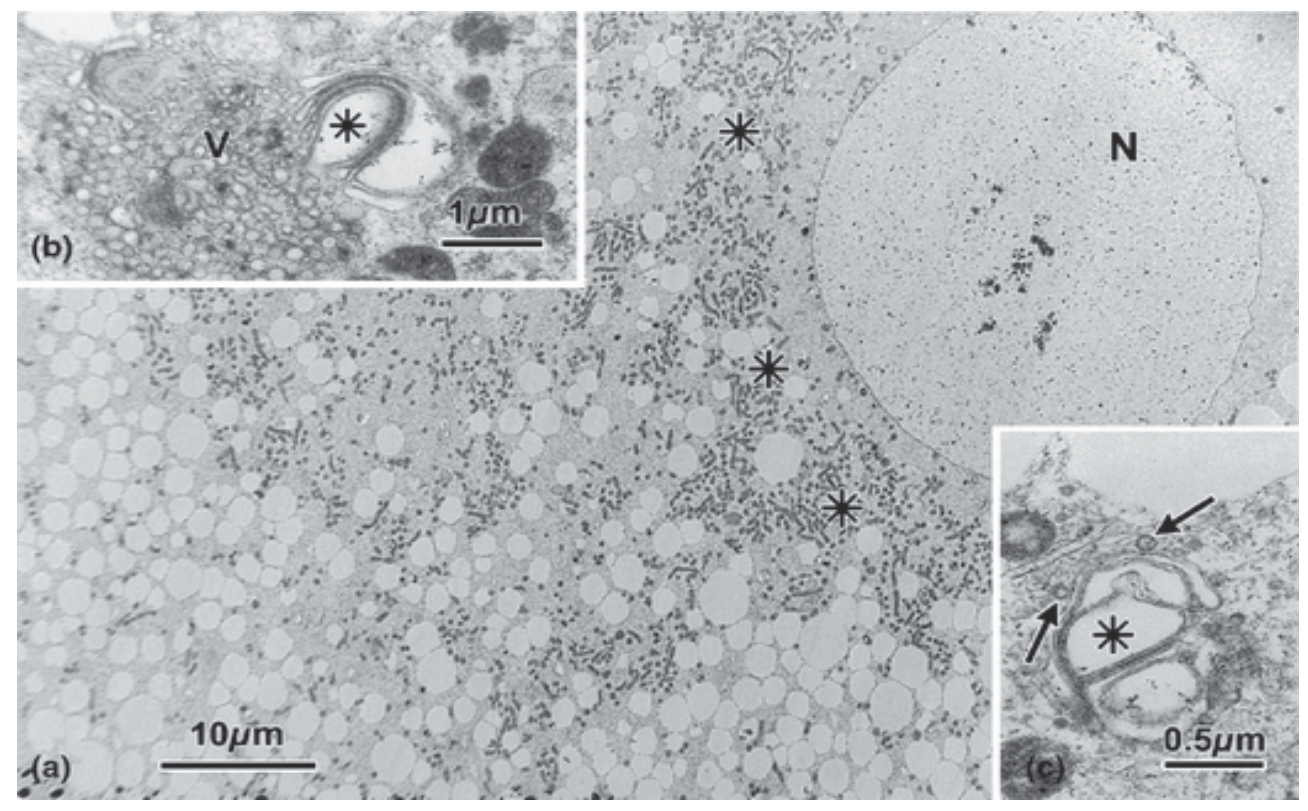

Fig. 2. (a) Balbiani's vitelline body (asterisks). N: nucleus. (b) One of several Golgi complexes (asterisk) with associated vesicles (V) in Balbiani's vitelline body. (c) Annulate lamellae (asterisk) with associated vesicles (arrows). 

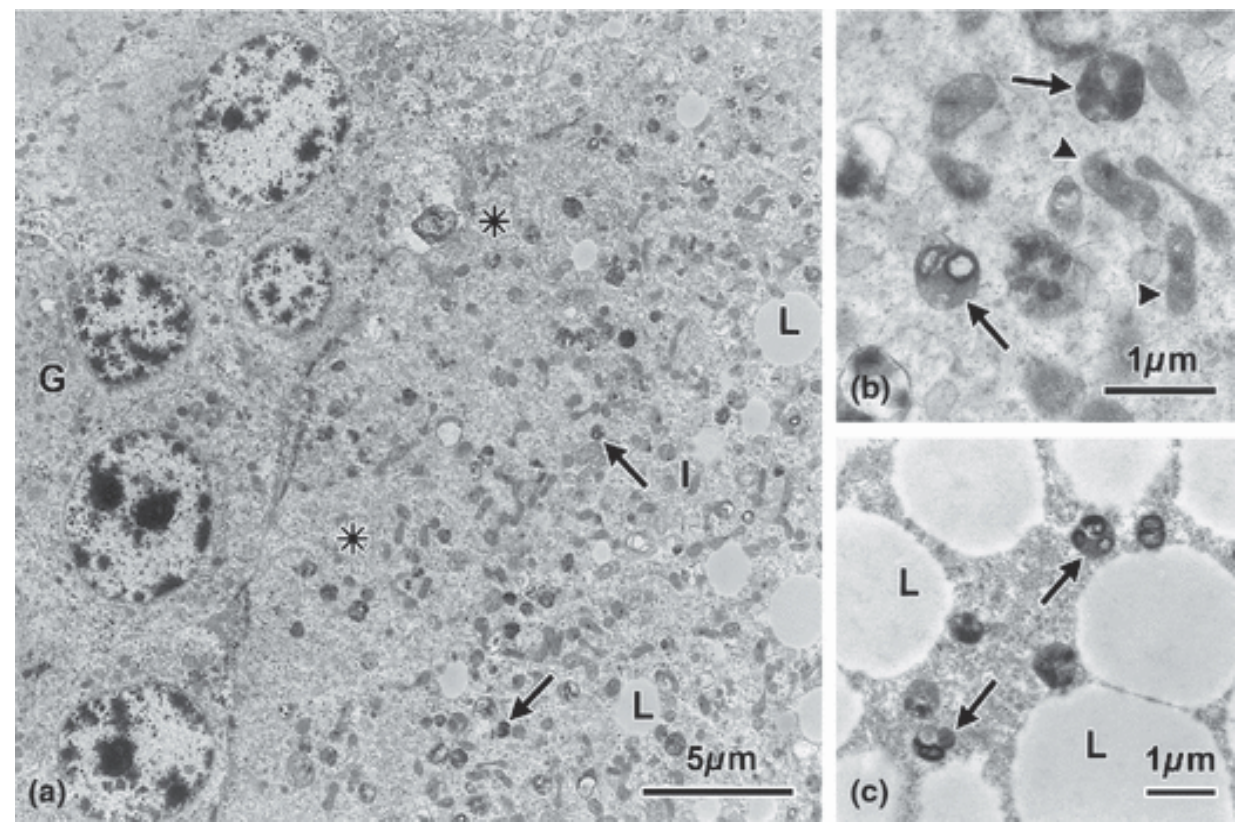

Fig. 3. (a) Large pre-vitellogenic follicle with a pseudo-stratified granulosa cell layer (G). The cortical (asterisks) and intermediate (I) regions of the ooplasm are shown. The intermediate region is characterized by the presence of numerous mitochondria, interspersed with lipid droplets (L) and small yolk spheres (arrows). (b) The cortical region of the ooplasm contains several small yolk spheres (arrows). Mitochondria (arrowheads) are observed between the small yolk spheres. (c) The central region of the ooplasm contains numerous lipid droplets (L) and relatively few small yolk spheres (arrows).
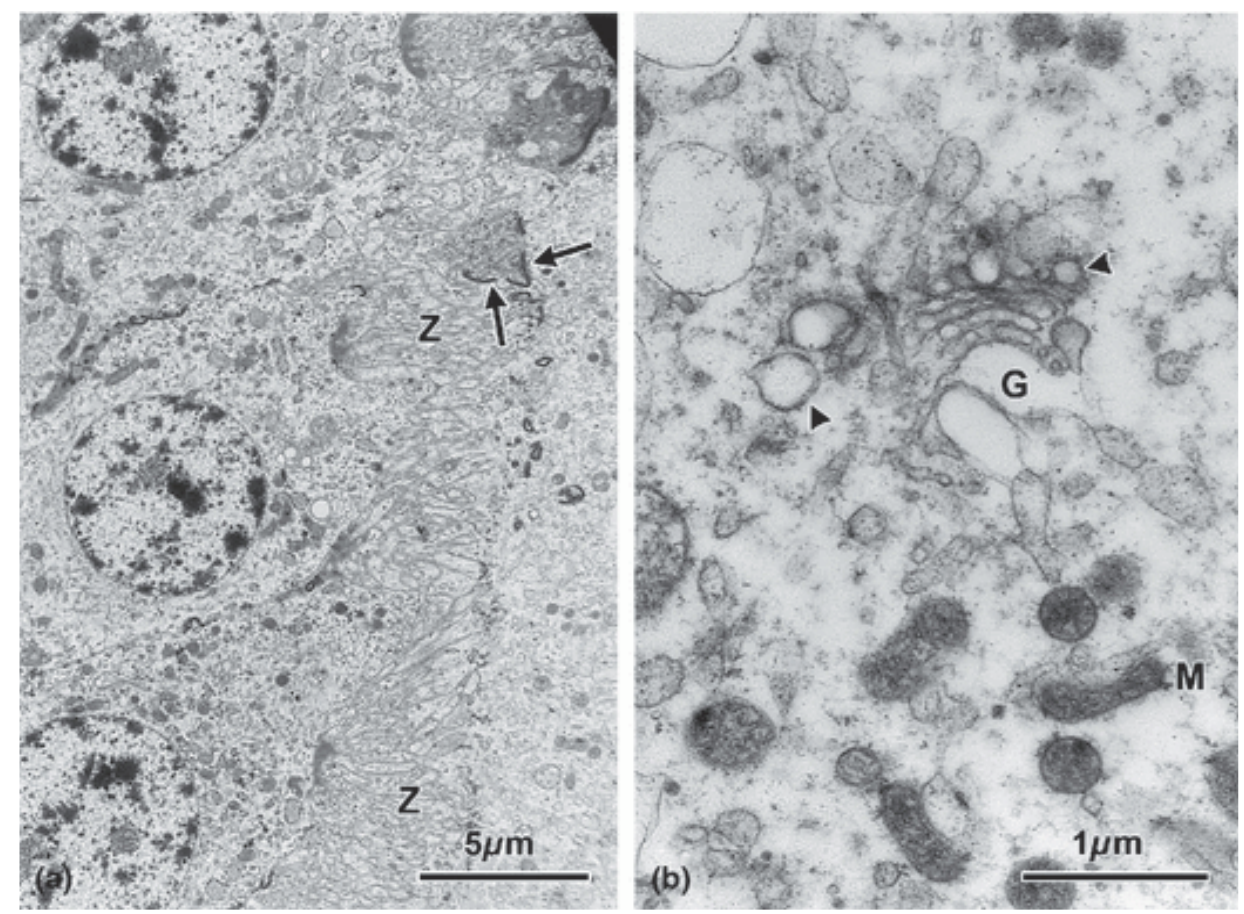

Fig. 4. Vitellogenic follicle. (a) Inter-digitations of the cell membranes of the oocyte and granulosa cells form the zona radiata $(Z)$. The granulosa cell membrane exhibits lining bodies (arrows). (b) Golgi complex (G), mitochondria (M) and vesicles (arrowheads) in the cortical region of the ooplasm. 

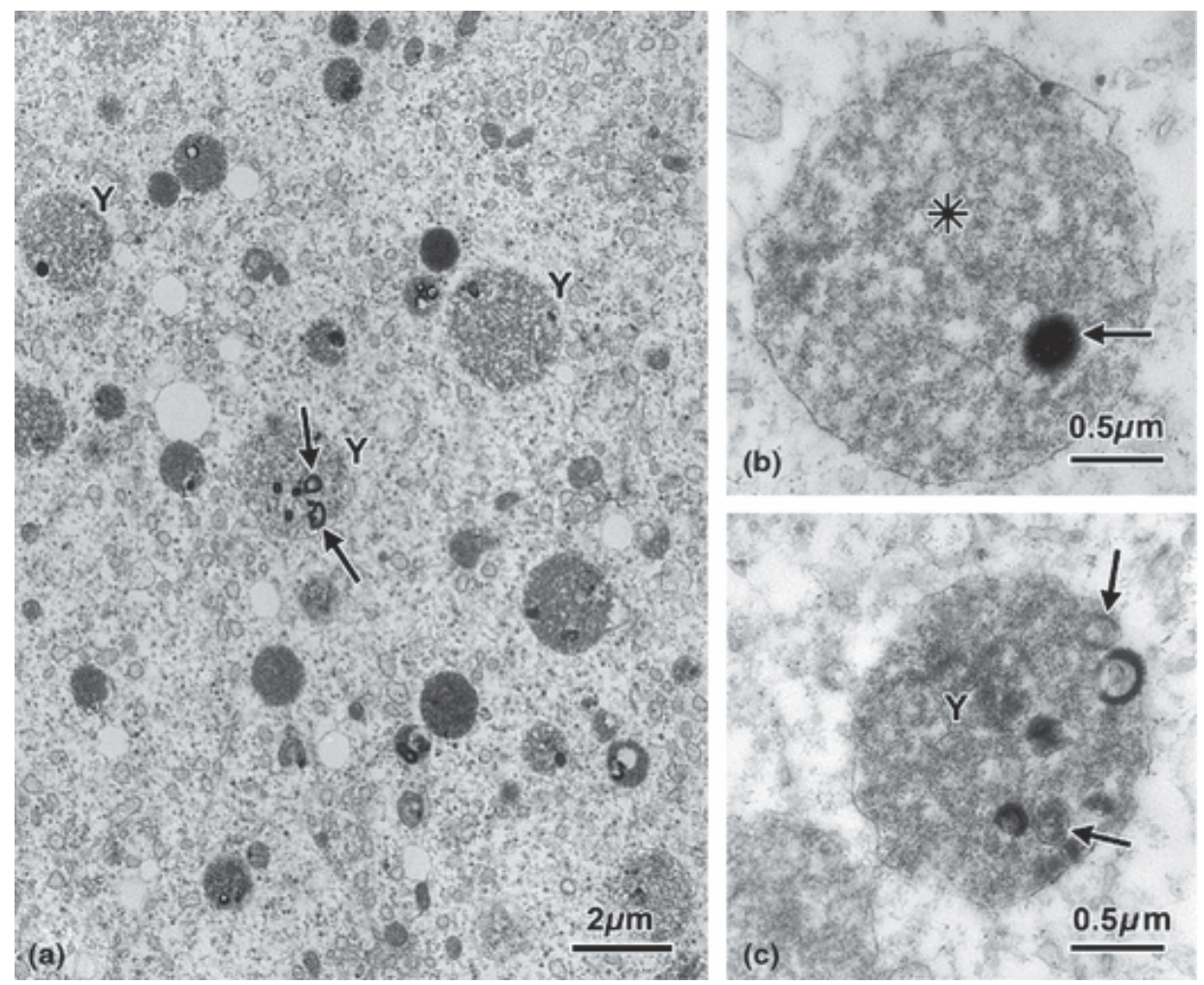

Fig. 5. Vitellogenic follicle. (a) Survey photomicrograph of the central region of the ooplasm. Numerous large yolk spheres $(\mathrm{Y})$ are present. Some yolk spheres contain lining bodies (arrows). (b) Large yolk sphere with an electron-dense yolk granule (arrow) surrounded by a finely granular material (asterisk). (c) Large yolk sphere $(\mathrm{Y})$ with disintegrating lining bodies (arrows). 